nurses so an appropriate and prompt plan of care can be implemented. Due to pressure on consultant Gastroenterology outpatient clinics, it is often difficult to see patients urgently who are acutely unwell. We recently evaluated referral pathways at the University Hospitals of Leicester (UHL) \& identified a number of patients who were admitted that could potentially have been managed as outpatients. As a result, of this finding we implemented two IBD Nurse-Led 'Hot Clinic's' per week.

Methods The criteria for referral were an established or new diagnosis of IBD with significant exacerbation of symptoms potentially requiring admission. On referral, the IBD nursing team made an initial phone assessment to confirm severity. All patients with on-going symptoms were then offered an appointment within 5 working days.

Results Over a 12-month period, 67 patients were referred to the Hot Clinic. 37 (55\%) self-referred via the IBD helpline, $11(16 \%)$ were referred from endoscopy with active disease (6 from the 2 week-wait pathway) and13 (19\%) direct from General Practice (GPs). 55 (82\%) had a face-to-face consultation. $3(5 \%)$ patients required admission to hospital and were admitted directly to a Gastroenterology ward; 2 (3\%) patients were treated with oral prednisolone but were admitted two weeks after the Hot Clinic review via Urgent care, and their admission was expedited to Gastroenterology within twentyfour hours. $2(3 \%)$ patients recently discharged following an admission contacted the helpline due to recurring symptoms and assessed in the Hot Clinic, and alteration in treatment prevented a readmission. A further 2 (3\%) patients admitted in the Medical Admissions Unit were assessed by the In-reach Gastroenterology team and were able to be discharged early with urgent review in a Hot Clinic appointment. Nine (13\%) patients were assessed over the phone, treatment was arranged which prevented a face-to-face consultation, and a plan of care was sent to both the patient and their GP. One patient declined an OPD and two never replied to calls or letters to contact the team.

Conclusion The introduction of ' IBD Hot Clinics' have allowed symptomatic patients with established IBD or newly diagnosed patients requiring urgent treatment, to be rapidly assessed by a specialist IBD nurse who can initiate appropriate treatments. This has shown to prevent admissions and fewer than $10 \%$ required admission in our cohort. The cost effectiveness of this service now requires further evaluation, with plans to expand this service.

\section{PTU-094 IS THE ROLE OF A GASTROENTEROLOGY ADVANCED NURSE PRACTITIONER VALUED?}

Anuska Glendinning*, David Walker. Royal United Hospitals Bath NHS Foundation Trust, Bath, UK

\subsection{6/gutjnl-2019-BSGAbstracts.453}

Introduction The Advanced Nurse Practitioner (ANP) is an established role within many hospitals and can be found in all aspects of health organisations. This new role has equipped nurses to take on more procedures as well as tasks traditionally associated with junior doctors, including the ability to diagnose and prescribe, whilst still retaining their foundation nursing roles. Previous research has focused on the ANP role within the community setting or emergency departments. This innovative study aimed to examine perceptions from the nursing and medical teams of an ANP working on a gastroenterology ward.

Methods A qualitative study was conducted at the Royal United Hospital Bath using three separate focus groups, each comprising of five gastroenterology doctors (FY1 to registrar), five gastroenterology nurses (grades -) and six ANP's. Each focus group lasted between 4-0 minutes and consisted of a set format of questions to guide discussions and aid with moderation of the group (eg. perceptions of the value of the ANP role within the ward environment, advantages of an ANP and how the ANP assists with a doctor's role). Transcripts of the interviews were analysed and the data was reduce into themes.

Results Thematic analysis identified three positive themes related to the perception of an ANP working of a gastroenterology ward; (1) Assisting with work load, (2) Team work and (3) Leadership. It was highlighted that due to the ANP's understanding of the processes of patient flow, tasks were preempted and performed in advance, improving efficiency and reducing the work load of other team members. Within the analysis there was an overriding theme of consistency; quotes from doctors included, "the ANP is an absolute 'life line' at the start of a new rotation. Their consistency allowed things not to get missed and acted as a safety net for us all, patients alike". There were several sub-themes relevant to gastroenterology particularly; 'sharing of applied skills and knowledge'. This was relevant for ascitic paracentesis, NG tube insertion, implementation of the liver care bundle and application of gastroenterology algorithms for complex patients which included GI bleeding.

Conclusions The study demonstrated that an ANP is a great asset to a gastroenterology ward and a valued member of the team. They provide consistency within the department, to both the nursing and medical teams. This is deemed vital, due to the ongoing four monthly rotations of junior medical staff, which is perceived as disruptive to team work and affecting consistency of patient care. The results have also identified how the ANP role appears to be more than just a 'work gap' solving role, they are paramount in sharing skills and knowledge with junior doctors as well as contributing to and enhancing team work, which is essential in health care transformation.

\section{PTU-095 UK QUALITATIVE FOCUS GROUP STUDY INVESTIGATING CURRENT PROVISION OF CARE FOR PEOPLE WITH MICROSCOPIC COLITIS}

${ }^{1}$ Annika Wilhelm, ${ }^{1}$ Christopher Hatton*, ${ }^{2}$ Matthew Kurien. ${ }^{1}$ Medical Affairs, Tillotts Pharma UK, Wellingore, UK; ${ }^{2}$ Academic Unit of Gastroenterology, Dept of IICD, University of Sheffield, Sheffield, UK

\subsection{6/gutjnl-2019-BSGAbstracts.454}

Introduction Microscopic colitis (MC) is a common cause of chronic, non-bloody watery diarrhoea that impacts healthrelated quality of life. No UK guidelines currently exist for MC. This has potential negative sequelae to patients as misdiagnoses and use of inappropriate treatments have been reported. This study examines UK provision of care for MC 
patients, with the ambition of developing a consensus document to support health care professionals in the future.

Methods This qualitative study involved four focus groups, and was conducted between March and October 2018. It involved a total of 27 expert participants IBD-specialist nurses $(n=8)$, gastroenterologists $(n=7)$, general practitioners $(n=5)$, colorectal surgeons $(n=2)$, pharmacists $(n=2)$, a histopathologist $(n=1)$ and clinical commissioning experts $(n=2)$. All participants were given pre-sessional information. Facilitated discussion was then undertaken between experts on key topic areas, and culminated in key themes, which were then further analysed.

Results The following themes were identified to influence patient care and outcomes: 1) Awareness. A lack of awareness particularly in primary care, and perceptions that MC is less common than conventional inflammatory bowel diseases were felt to be factors influencing diagnosis and management. 2) Symptom overlap with IBS. As symptomatology for both MC and IBS are similar, the expert group felt referrals to secondary care are likely to be restricted, leading to increased GP visits, enhanced use of over the counter medications, and diminished quality of life. 3) Faecal calprotectin (FC). The expert group felt referral practice from primary care was too reliant on FC as a discriminator, as active MC can have FC levels below the conventional referral thresholds. 4) Biopsies. The expert group identified that the diagnosis of MC may be missed as endoscopists neglect to take colonic biopsies, either through a lack of awareness, or determined by time and cost restrictions. 5) Treatment. This theme demonstrated significant variations in clinical practice, with an array of therapeutic interventions used to manage MC. Clinicians frequently lacked awareness of MC treatments and were not aware that budesonide is the only established treatment, as evidenced in randomised-controlled trials.

Conclusions This is the first study to examine service provision for MC in the UK. Key themes have been identified through expert opinion, demonstrating why optimal diagnosis and management of $\mathrm{MC}$ has been difficult to operationalise in the UK. These findings will be used to develop a consensus document that has benefits to healthcare professionals and patients.

\section{PTU-096 INTRODUCTION OF A GASTROENTEROLOGY OUTREACH SERVICE AND ITS IMPACT WITHIN A TERTIARY UNIVERSITY HOSPITAL}

Timothy Keen, Imdadur Rahman, Philip Boger, Nicholas Coleman, Bernard Stacey. University Hospital Southampton, NHS Foundation Trust, Southampton, UK

\subsection{6/gutjnl-2019-BSGAbstracts.455}

Introduction Hospital services are under increasing pressure which has led to an increased drive for earlier discharges. Various initiatives have begun to manage this. Within a UK tertiary hospital, a gastroenterology outreach team was formed from a pre-existing inpatient team alongside 2 remaining inpatient gastroenterology teams. This change coincided with the introduction of a new IT system with electronic triages and referrals. The aim of this service evaluation was to assess the impact on patient management, hospital stay and referring specialty satisfaction.

Methods The outreach team consisted of a consultant gastroenterologist and either a nurse specialist or junior doctor. The role of the outreach team was to review direct to gastroenterology triages within an acute medical admissions unit as well as all ward referrals. The direct to speciality triages were managed on the acute medical unit by the outreach team or admitted to a ward to be cared for by one of the inpatient teams. A retrospective analysis of a prospective kept database of all speciality triages and referrals was performed over a 15 month period from October 2017 to January 2019.

Results A mean increase of $15 \%$ per year in the number of direct to speciality triages and referrals was noted (mean 86/ month). $49 \%$ of referrals were from 3 specialities; cardiology $(25 \%)$, surgery (12\%) and respiratory (12\%). 100\% of referrals and speciality triages were seen within 24 hrs. Vomiting and/or dysphagia (33\%), diarrhoea (16\%), GI bleeding (14\%) and weight loss (12\%) account for the majority of speciality triages (mean 68/month). The outreach team managed 22\% of these without input from any inpatient teams. Following the introduction of the outreach team there has been a $14 \%$ reduction in the number of patients admitted to a ward from the acute medical unit (67\% to $53 \%)$, an $11 \%$ increase in patients discharged $<48$ hours ( $34 \%$ to $45 \%$ ) and a reduced length of hospital stay by 2 days (median of 4 to 2 days). A 1632 bed days saved per year equated to a cost saving of $£ 652800$ annually. Inpatient teams rated the outreach team as $9.2 / 10$ overall and at $8.7 / 10$ for patient management $(1=$ poor, $10=$ excellent).

Conclusions The outreach team has been successful in improving initial patient management which has subsequently reduced the number of speciality triages admitted to a ward, increased patient discharges $<48$ hours, reduced length of stay, saved bed days and increased satisfaction with the service. We believe if this model of patient care was introduced to other hospitals it will have similar benefits.

\section{PTU-097 LEICESTER CITY CCG EXPERIENCE: DO DIRECT PHONE CALLS TO NON-RESPONDERS IMPROVE BCSP UPTAKE?}

Hui Lin Lee*, Racheal Fenton, Shelley Mellor, Karen Emery-Downing, Richard Robinson. University Hospitals Leicester, Leicester, UK

\subsection{6/gutjnl-2019-BSGAbstracts.456}

Introduction Bowel Cancer Screening Programme (BCSP) has shown to be associated with a substantial stage shift in colorectal cancer diagnosis. In 2015/16, the overall percentage uptake for gFOBt bowel screening nationally was $56.4 \%$. There was a lower uptake across Leicester City CCG of $45.8 \%$. The aim of this project was to assess if direct phone calls to non-responders towards gFOBt bowel screening improve BCSP uptake locally.

Methods We identified individuals from 8 local GP surgeries who had not returned their BCSP gFOBt kit between September and October 2016. These individuals were all phoned by a nurse specialist. During the telephone call encounter, we discussed the importance of bowel cancer screening, checked if each subject had received the 1 st gFOBt kit, identified if there was any language barrier, offered further information (written/ picture booklets) and enquired if subjects would like another kit sent out again.

Results A total of 412 (202 females, 210 males) non-responders were identified. The mean and median age of the subjects was 60 years and 64 years respectively. A total of 580 phone 